\title{
NATURALISMOS Y ACUMULACIÓN POR DESPOSESIÓN: PARADOJAS DEL DESARROLLO SUSTENTABLE
}

\author{
Carlos Santos \\ Universidad de la República - Uruguay
}

Resumen: Este artículo analiza la manera en que dos procesos globales (el acaparamiento de tierras y las políticas de conservación de la naturaleza) intervienen en las dinámicas de reproducción social de los habitantes del entorno de un parque natural en Uruguay. Ambas escalas permiten poner en juego los diferentes naturalismos categorizados por Descola. La idea de acumulación por desposesión, de Harvey da cuenta de esta fase de expansión del capitalismo sobre los "bienes comunes", cuya característica central es la degradación y depredación de los recursos naturales. Por su parte la implementación de políticas públicas de conservación en este contexto consolida paisajes duales (unos dedicados a la preservación de la naturaleza y otros a su explotación indiscriminada). El análisis permite discutir la insustentabilidad de las relaciones sociales dominantes, así como las diferentes dimensiones y escalas de análisis complejizan la comprensión de los efectos locales y combinados de estos dos fenómenos globales.

Palabras clave: acumulación por desposesión, antropología ambiental, conservación, sustentabilidad.

Abstract: This paper examines how two global processes (land grabbing and conservation policies) are involved in the dynamics of social reproduction of the inhabitants of a national park in Uruguay. Both scales can be brought into play the different naturalisms categorized by Descola. Harvey's idea of accumulation by dispossession, realizes this phase of expansion of capitalism on the "commons" whose central feature is the degradation and natural resource depletion. Meanwhile the implementation of

* Una versión inicial de este trabajo fue expuesta en el panel Problemas globais e conflitos locais del Seminario Internacional de Ciencias Sociales-Ciencia Política “Buscando o sul” de la Unipampa (Universidad Federal do Pampa), Campus de São Borja. Agradezco al Prof. Daniel Etcheverry y a los colegas participantes por la invitación y los comentarios recibidos.

Horizontes Antropológicos, Porto Alegre, ano 20, n. 41, p. 331-356, jan./jun. 2014 http://dx.doi.org/10.1590/S0104-71832014000100012 
conservation policies in this context consolidates dual landscapes (some dedicated to the preservation of nature and others to indiscriminate exploitation). The analysis allows us to discuss the unsustainability of the dominant social relations, as well as various sizes and scales of analysis bring complexity of the understanding of local and combined effects of these two global phenomena.

Keywords: accumulation by dispossession, conservation, environmental anthropology, sustainability.

\section{Introducción: la escala local del acaparamiento de tierras y el conservacionismo}

Los albores del siglo XXI han sido acompañados por dos procesos de apariencia contradictoria: el aumento global de la producción de bienes y la preocupación por la conservación de los recursos naturales. Sin embargo los efectos de estos procesos se retroalimentan en detrimento de las condiciones de reproducción social de sectores subalternos, replegados a zonas que hasta ahora habían sido consideradas ‘marginales’ para el desarrollo de los sistemas productivos.

Los efectos más evidentes de estos procesos son el acaparamiento de tierras por un lado (conocido en inglés como land grabbing), y el avance de las políticas de conservación de la naturaleza (como el establecimiento formal de áreas protegidas), por otro. Ambos son exponentes de lo que Philippe Descola ha denominado "naturalismo", o sea expresiones de la relación alienada de la naturaleza propias de las sociedades occidentales. La primera bajo la forma de un naturalismo “depredador” y la otra bajo un naturalismo “conservacionista”.

En este artículo se analizan los impactos que estos dos procesos de escala global producen en un espacio en particular. A partir de la implementación del Parque Nacional Esteros de Farrapos e Islas del Río Uruguay ${ }^{1}$ y del análisis del proceso de expansión del agronegocioº en el departamento de Río Negro

\footnotetext{
1 El análisis del proceso de implantación de esta política de conservación surge de la tesis de maestría del autor (Santos, 2011) en el marco del programa de posgrado en Ciencias Sociales de UNGS-IDES, Beca “Naturaleza, sociedad y territorio” del Programa Regional de Becas del Consejo Latinoamericano de Ciencias Sociales CLACSO-ASDI 2008 para América Latina y el Caribe).

2 Este análisis tiene como antecedente el trabajo "La expansión del agronegocio agrícola en Uruguay: impactos, disputas y discursos” en coautoría con Gabriel Oyhantçabal e Ignacio Narbondo, discutido en el panel "Family farming and agribussines: Territorial disputes and symbolic struggles" del Latin America Studies Association (LASA) Congress (San Francisco, 2012).
}

Horizontes Antropológicos, Porto Alegre, ano 20, n. 41, p. 331-356, jan./jun. 2014 
(litoral oeste de Uruguay), se pudo apreciar el proceso de deterioro en las condiciones de vida de los pescadores, apicultores, cazadores y productores familiares del entorno de esta área protegida.

\section{La preocupación global por la protección de la naturaleza}

La expansión de las áreas protegidas en los últimos años puede entenderse como parte del proceso de globalización; la cuestión ambiental es una de las primeras preocupaciones necesariamente no locales de la sociedad, los estados y la institucionalidad internacional lo que ha tenido como contraparte la identificación de la biodiversidad como uno de los primeros objetos de la preocupación global sobre la naturaleza.

En los últimos cuarenta años a lo largo y ancho de todo el mundo se ha producido una verdadera explosión de áreas naturales protegidas establecidas formalmente. Mientras que en la década del sesenta en todo el planeta había poco más de 1.000 áreas protegidas oficiales, en 2006 el número llegaba a más de 108.000. Esta expansión tuvo su punto de inflexión en la Convención sobre Biodiversidad de Naciones Unidas (que formó parte de los acuerdos de la Cumbre de la Tierra). Allí se estableció el acuerdo de proteger al menos un $10 \%$ de la superficie global, habiéndose superado el 12\%, más de 30 millones de kilómetros cuadrados (Dowie, 2006).

Las áreas protegidas o parques naturales existen en Uruguay desde mediados del siglo XX, pero su incorporación dentro de un Sistema Nacional de Áreas Protegidas (SNAP), gestionado desde el Estado central es una novedad de comienzos del siglo XXI.

Entendidas como parte de una estrategia de desarrollo sustentable, las áreas naturales protegidas implican la opción por un determinado modelo de desarrollo. Esta opción es válida tanto cuando la declaración de área protegida para un territorio determinado es adoptada por las autoridades ambientales, por las comunidades que viven en el territorio o en su entorno, o aún en la definición de actores académicos o no gubernamentales aplicados a la conservación de recursos naturales. Sin embargo tal opción no siempre es explícita ni manifiesta en el mismo grado para todos los actores involucrados.

Las áreas naturales protegidas tienen implicaciones sociales, entre otras, la aparición de nociones sobre ambiente y naturaleza, en lugares donde no necesariamente existían como tales hasta hace muy poco tiempo. Por otra parte,

Horizontes Antropológicos, Porto Alegre, ano 20, n. 41, p. 331-356, jan./jun. 2014 
la participación social, recomendada, reclamada y asumida, muchas veces es vista como un fin en sí misma, como una etapa necesaria en la implementación de las áreas protegidas, sin cuestionar sus procesos, criterios u objetivos.

\section{La presión global sobre la tierra}

El proceso definido como "acaparmiento mundial de tierras” es en términos concretos el nombre que recibe "la actual explosión de operaciones comerciales (trans)nacionales de tierras que giran en torno a la producción y venta de alimentos y biocombustibles” (Borras Jr.; Franco, 2010, p. 2). Este término ha sido acuñado para denunciar los impactos de este proceso global sobre la vida de los campesinos y agricultores familiares, quienes sufren en concreto los efectos de esta dinámica global de concentración.

Esta nueva fase de expansión del capital -conducida por el capital financiero a nivel trasnacional- es una suerte de respuesta o salida a las crisis generadas por la especulación, a partir de una necesidad de anclar la riqueza en los recursos naturales. En términos de Borras Jr. y Franco (2010, p. 4) "la confluencia de las diversas crisis ha desembocado en una revaloración de la tierra, que apunta hacia un incremento significativo de su valor económico”. Solamente en el período comprendido entre 1990 y 2005, la tierra cultivada de todo el planeta aumentó 2,7 millones de hectáreas al año (The World Bank, 2010, p. x). El propio Banco Mundial -responsable de las políticas financieras y de desarrollo que han desencadenado estas dinámicas en las últimas décadasha advertido la disparidad de este aumento en los países del Tercer Mundo:

el deterioro en los países industrializados y de transición (de -0,9 y -2 millones de hectáreas, respectivamente) fue más que compensado por incrementos de 5,5 millones de hectáreas por año en los países en desarrollo. La expansión de la tierra cultivable, que habría sido mucho más amplia sin los aumentos en productividad, se concentró en el África subsahariana, América Latina y el sudeste de Asia. (The World Bank, 2010, p. x, traducción mía).

Los conductores de del acaparamiento de tierras, han sido la producción de aceites vegetales, la caña de azúcar, el arroz, el maíz y las plantaciones forestales. Particularmente sobre América Latina, el cultivo de soja desde la década del 90 y más recientemente los cultivos de árboles han dinamizado una 
reforma agraria al revés, que ha conducido a la concentración de la propiedad de la tierra en detrimento de campesinos y agricultores familiares.

El avance sobre las tierras cultivables de los países del Tercer Mundo ha implicado no solamente una modificación de los rubros de acción del capital financiero, sino un aprovechamiento diferencial de las capacidades productivas de estos países, en beneficio de los inversionistas.

Es necesario identificar los actores que están detrás de este proceso de acaparamiento de tierras. Oyhantçabal y Narbondo (2011, p. 113) sostienen que

Los inversores provienen de todas parte del mundo, aunque es cada vez más relevante el interés de las potencias emergentes asiáticas China e India, que hasta 2009 acumulaba inversiones en Latinoamérica por U\$S 22.000 millones en toda su historia, creció exponencialmente durante 2010 con el anuncio de nueves grandes operaciones por casi U\$S 17.000 millones. Por su parte, de las diecinueve inversiones chinas en Latinoamerica desde 2005, quince se destinaron a la producción de materias primas.

En el caso de América del Sur, algunas empresas de origen brasileño han reproducido está dinámica global de acaparamiento sobre los demás países de la región, lo que obliga a pensar no solamente en la nacionalidad de los 'apropiadores' sino en el papel que juegan en la cadena global de un agrocomplejo de producción de alimentos y energía.

\section{De lo global a lo local: un área protegida en un enclave de agronegocio}

El espacio local donde pretendemos aterrizar estas discusiones (la confluencia de las políticas de conservación y el acaparamiento de tierras) es el Parque Nacional Esteros de Farrapos e Islas del Río Uruguay, ubicado en el departamento de Río Negro, en el litoral oeste de Uruguay. Uno de los principales elementos a tomar en cuenta para la elección de esta área como objeto de estudio es el hecho de que se trata de una de las pocas áreas nuevas en el proceso de incorporación al SNAP, ya que la mayoría de las que se encuentran proyectadas o han ingresado ya contaban con un carácter previo de conservación o protección (por ejemplo a nivel municipal). Por otra parte, Farrapos es un área que ingresó al SNAP a fines de 2008 y aún se encuentra en proceso de implementación. El área se puede caracterizar como un humedal con islas fluviales que se ubica en ambas márgenes del Río Uruguay. Este humedal se 
extiende desde la ciudad de Concepción (en la margen argentina), mientras que a la altura de la localidad de San Javier el humedal cruza a la margen uruguaya, extendiéndose en ambas márgenes hasta la altura de la ciudad de Fray Bentos.

El área delimitada como protegida abarca una superficie de 6.327 hectáreas, que actualmente son propiedad del Ministerio de Vivienda, Ordenamiento Territorial y Medio Ambiente (desde agosto de 2001, mientras que su anterior propietario era el Ministerio de Ganadería, Agricultura y Pesca, a cargo del Instituto Nacional de Colonización). Como plantea Diegues (2005, p. 39) para el caso de Brasil, el área de Farrapos fue delimitada estrictamente en base a criterios científicos; "los denominados 'atributos naturales de los ecosistemas' definidos por la biología, ecología no humana, son considerados los únicos criterios ‘científicamente’ válidos para administrar el espacio y los recursos naturales".

El interés para la conservación de estos esteros es su carácter de representatividad de los humedales de zonas transicionales entre áreas tropicales y húmedas, su papel en la regulación hidrológica del río Uruguay y su carácter transfronterizo (con Argentina), además de una serie de especies particulares de flora y fauna.

\section{La producción (global) de un área protegida (local)}

Siempre los eventos que se suceden en un determinado espacio están afectados por dinámicas que le trascienden. Pero este escenario de la ruralidad y la conservación globalizadas plantea ejemplos particulares de cómo la vida cotidiana de un territorio específico está limitada por eventos transnacionales. Por ejemplo, ¿qué tienen en común la ciudad iraní de Ramsar, a orillas del Mar Caspio, con la ciudad suiza de Montreax a orillas del lago del lago Lemán, con los Esteros de Farrapos, a orillas del río Uruguay? En principio, claro, las tres están ubicadas sobre importantes cursos de agua. Pero más allá de esta evidente y rebuscada conexión, algunos de los procesos por los que ha pasado la zona de Farrapos están directamente vinculados con acontecimientos que sucedieron en Ramsar y Montreux, como veremos a continuación.

En el año 2000, el Ministerio de Vivienda Ordenamiento Territorial y Medio Ambiente adquirió un predio de 6.327 hectáreas en el Departamento de Río Negro, que comprende la zona de los llamados Esteros de Farrapos.

Horizontes Antropológicos, Porto Alegre, ano 20, n. 41, p. 331-356, jan./jun. 2014 
Anteriormente el predio pertenecía al Ministerio de Ganadería, Agricultura y Pesca y era gestionado por el Instituto Nacional de Colonización. El objetivo de este traspaso fue la incorporación de Farrapos a al Sistema Nacional de Áreas Protegidas que había sido aprobado por ley ese mismo año.

En el año 2004 a iniciativa de la Dirección General de Recursos Naturales Renovables del Ministerio de Ganadería, Agricultura y Pesca (DGRNR), los Esteros de Farrapos y dos islas del Río Uruguay ubicadas al sur de los Esteros, fueron presentadas como sitio a ser incluido en la Convención Ramsar, la Convención de Naciones Unidas para la protección de humedales. Precisamente la DGRNR era en ese entonces el punto focal del Estado uruguayo para esta Convención.

El sitio Ramsar tiene una extensión de 17.496 hectáreas, 6.917 corresponden a islas del Río Uruguay, 6.972 corresponden al Estero de Farrapos y 3.607 corresponden a la superficie del espejo de agua del Río Uruguay (DGRNR-MGAP, 2004).

Además del propio valor para la conservación que presentan los Esteros de Farrapos, otra situación incidió en que el Estado uruguayo los postulara para su inclusión en la Convención Ramsar, y esto tiene que ver con el llamado Registro de Montreax.

Este registro es una suerte de lista negra de todos aquellos países que habiendo ratificado el Convenio e incluido sitios bajo su amparo no han cumplido con las pautas de conservación establecidas como compromiso.

Uruguay ratificó el Convenio de Ramsar en 1971. A fines de la dictadura militar (en 1984) el Estado uruguayo incluyó los Bañados del Este como sitio a ser contemplado dentro del Convenio. Sin embargo, también como herencia de la dictadura, se permitió "la existencia de áreas de humedales intervenidas y abocadas a agricultura bajo riego (arroz)” (DGRNR-MGAP, 2008, p. 29), lo que hizo que en 1990 la Conferencia de las Partes del Convenio de Ramsar pusiera en marcha este Registro de Montreux y los Bañados del Este de Uruguay quedaran incluidos en él. La "intervención” que el gobierno militar permitió sobre los Bañados del Este, afectó a una casi la cuarta parte del sitio Ramsar (o sea, unas 100.000 hectáreas del sitio total de 400.000). Para salir del Registro de Montreux, el Estado uruguayo debe incluir sitios por una superficie equivalente a esas 100.000 hectáreas.

La importancia del Registro de Montreaux no tiene que ver solamente con una cuestión de “imagen” del Estado: quienes están en este registro ven

Horizontes Antropológicos, Porto Alegre, ano 20, n. 41, p. 331-356, jan./jun. 2014 
estrictamente limitada su aspiración a la postulación de fondos internacionales para la conservación de estos sitios.

La "estrategia nacional” para "sacar el Sitio Bañados del Este y Franja Costera del Registro de Montreux" consiste en "tomar las medidas necesarias para revertir la situación de la superficie que decidió la ubicación del sitio en el Registro y paralelamente, compensar esa superficie (aproximadamente 96 mil hectáreas) con la creación de nuevos sitios” (DGRNR-MGAP, 2008, p. 29).

Dentro de esos nuevos sitios, están los Esteros de Farrapos: un sitio “compensatorio” de la zona de los Bañados del Este, humedales que fueron incluidos en la Convención pero cuya conservación no fue debidamente atendida por el Estado (que se había comprometido a ello al ponerlos al amparo de la Convención).

En el año 2008, se realizó la audiencia pública y se incorporó Esteros de Farrapos al SNAP, bajo la categoría de "Parque Nacional”, constituyéndose en la segunda área en ingresar al sistema (la primera había sido unos meses antes Quebrada de los Cuervos en el departamento de Treinta y Tres). El área que se declara como área protegida e ingresa al SNAP corresponde a los Esteros de Farrapos, la región continental del sitio Ramsar y no incluye -al menos en esta etapa- las islas del río Uruguay, las que ingresarían en una etapa posterior. En el año 2009 el SNAP designó un encargado del área y en 2010 se ha conformado un equipo con tres trabajadores de campo para el área (guardaparques y guarda-islas) al tiempo que comenzó a funcionar la Comisión Asesora Específica (CAE) de Esteros de Farrapos e Islas del Río Uruguay (Boletín SNAP, 2010). En 2103 aún continúa en proceso de elaboración el Plan de Manejo del área que establecerá los límites del área de amortiguación así como las actividades permitidas y su distribución dentro del parque nacional y su entorno.

\section{Sobre intensificación agrícola y desigualdades sociales}

Por otro lado, hemos sostenido previamente que la tendencia histórica hacia la concentración de los medios de producción (particularmente, la tierra) ha afectado las condiciones de reproducción social de los habitantes del campo. Esto no es una novedad de los procesos novedosos como el acaparamiento de tierras. Lo novedoso es la intensidad del fenómeno y el carácter central 
que ocupa la dimensión ambiental en el establecimiento de las desigualdades sociales: los excluidos de la tierra son también ahora perjudicados en las condiciones del entorno al cual fueron marginados para subsistir.

A partir de la intensificación y la transformación de la producción agrícola desde la década del sesenta ha habido un proceso de concentración de población en los centros urbanos en detrimento de la zona rural. La población que ha migrado a los centros urbanos ha transformado sus dinámicas de reproducción social. En muchos casos, trabajadores rurales provenientes de la ganadería o la agricultura han adaptado sus dinámicas a la realización de actividades extractivas, como la pesca, la recolección o la caza, o la especialización en actividades productivas como la apicultura. Las dos localidades urbanas ubicadas en el entorno del área protegida son San Javier (al norte) y Nuevo Berlín (al sur) ambas en el departamento de Río Negro. La mayor parte de los habitantes de estas dos localidades alternan entre sus actividades entre la pesca, apicultura y caza, de forma complementaria y estacional. La temporada de mayor concentración de la actividad de pesca se da entre los meses de abril a octubre, mientras que la apícola se concentra entre los meses de noviembre a marzo. La caza se realiza a lo largo de todo el año. Sólo en Nuevo Berlín, actualmente unas 300 familias alternan entre estas actividades, lo que les otorga un carácter fundamental para la reproducción social.

Particularmente en la zona que tiene como epicentro la localidad de San Javier y se extiende en la zona norte del Estero, la actividad que se realiza primordialmente es la cría de ganado, que podemos clasificar en dos modalidades de acuerdo al régimen de tenencia de la tierra: un grupo está integrado por quienes poseen establecimientos rurales en la zona y que utilizan el Estero como zona de pastoreo, mientras que el otro está conformado por ganaderos sin tierra, que realizan otras actividades (asalariados rurales, empleados) y complementan sus ingresos con la cría de ganado. La situación de estos productores ganaderos sin tierra es una consecuencia directa del aumento en el precio de la tierra (en este caso, del arrendamiento) debido al proceso de concentración conducido por la intensificación de los cultivos de soja y forestales. En el predio de Esteros de Farrapos existe un número aproximado de 3.000 cabezas de ganado vacuno, en las condiciones descriptas.

La intensificación de la producción agrícola y su impacto en el precio de la tierra (tanto en la venta como en el arrendamiento) ha tenido una incidencia directa en las formas de subsistencia de estos amplios sectores de las 
localidades de Nuevo Berlín y San Javier, al tiempo que ha implicado profundos cambios en el mundo del trabajo rural.

El territorio que actualmente ocupa el área protegida y su área adyacente tiene una larga historia de usos, que van desde la ocupación estacional para la caza, la pesca y la recolección en los tiempos anteriores a la conquista, a una creciente intensificación de la agricultura.

También en un lugar muy próximo a esta zona que ahora conocemos como Esteros de Farrapos, dio inicio el proceso de colonización -a comienzos del siglo XVII- con la introducción del ganado vacuno, que ocuparía el territorio al norte del Río Negro antes que los propios colonizadores europeos.

Gestionada como una de las vaquerías de las Misiones Jesuíticas, una de las formas iniciales de apropiación tiene que ver con el reclamo de propiedad privada de estos territorios, que tuvo lugar a mediados del siglo XVIII. La definición de la propiedad privada de la tierra ha sido el organizador de la vida social y productiva de Uruguay desde entonces.

Algunos se establecieron como dueños de la tierra y otros como trabajadores de las grandes estancias ganaderas. A fines del siglo XVIII y comienzos del siglo XIX la zona comenzó a orientarse hacia la producción agrícola, a partir de la llegada de colonos europeos pero esta vez procedentes de Alemania o el Cáucaso. A partir de aquí, a lo largo del siglo XX se daría un proceso de intensificación agrícola en todo el departamento de Río Negro. A finales del siglo XX y en los primeros años del siglo XXI, este proceso se intensificaría aún más a partir del auge de la producción forestal y sobre todo de la producción de soja.

Veamos en detalle algunos elementos de los procesos desencadenados en los últimos años. Macadar y Domínguez (2008, p. 84) sostienen que la consolidación del modelo productivo agroexportador condujo a la concentración de la población “en el sur y litoral oeste del país” haciendo que éstas áreas fueran "las más dinámicas por su continua atracción de capital y mano de obra en detrimento del centro y norte del país”. A nivel interno del departamento de Río Negro, el proceso de consolidación de este modelo productivo condujo a la concentración de la propiedad de la tierra y al desplazamiento de los pequeños productores agropecuarios (en general, de carácter familiar, algunos propietarios otros arrendatarios) desde el medio rural a las pequeñas ciudades.

En términos de evolución de la población, en el año 1956 el departamento de Río Negro tenía una población rural de 14.729 personas, mientras que en

Horizontes Antropológicos, Porto Alegre, ano 20, n. 41, p. 331-356, jan./jun. 2014 
1966 ascendía a 10.640. En ese mismo período se redujo a la mitad el número de trabajadores rurales, pasando de un promedio de 6 trabajadores por predio en 1956 a 3,2 trabajadores por predio en 1966 (Nuestra Tierra, 1970).

De acuerdo al censo de 1963, la población rural representaba el 34,4\% de la población del departamento, mientras que las localidades de Nuevo Berlín y San Javier tenían una población de 1.912 (Nuevo Berlín) y 1.178 habitantes (San Javier). En 2004 el peso de la población rural se redujo a 12,5\%, el peso relativo de las localidades de Nuevo Berlín y San Javier se mantuvo, mientras que el peso de la capital departamental (Fray Bentos) aumentó de 37,4\% en 1963 a 42,8\%. En términos de migración interdepartamental, Macadar y Domínguez (2008) ubican a Río Negro como uno de los departamentos expulsores de población entre 1996 y $2001 .^{3}$

\section{El protagonismo del agronegocio}

La zona donde se encuentran los Esteros de Farrapos no ha sido ajena al avance de la frontera agrícola ni a las recientes transformaciones que ese avance ha implicado, ante la consolidación del agronegocio como actor fundamental en la producción rural, lo que Hernández (2009, p. 39) ha denominado "ruralidad globalizada". El concepto de agronegocio supone una superación de la visión de la producción agrícola acotada a la explotación, postulando la integración horizontal y vertical de la producción agrícola e industrial, para lo cual es necesario superar también la idea de las fronteras nacionales (Hernández, 2009).

Lo que se denomina área de amortiguación (área próxima al área protegida, pero no incluida dentro de sus límites ${ }^{4}$ ) incluye una zona de intensa producción agrícola y forestal (ya que la ganadería ha ido cediendo paso a estas otras producciones). En este proceso, la principal transformación ha sido la transnacionalización de la producción agrícola con la emergencia de empresas

\footnotetext{
3 Según los autores, el saldo migratorio negativo que presenta el departamento de Río Negro en ese período (sobre todo en relación con el vecino departamento de Paysandú) se revierte a partir del año 2003 cuando empieza el proceso de construcción de la actual fábrica de celulosa de UPM/Botnia en Fray Bentos (que según diferentes estimaciones, ocupó unas 3.000 personas durante el proceso de construcción de la planta).

4 Hasta ahora sólo se conoce la delimitación del área protegida y no la del área de amortiguación o área buffer.
} 
que compran tierras (en el caso de la forestación) o las arriendan (en el caso de la soja) en grandes extensiones, aplicando paquetes tecnológicos “de diseño”, cuyo ejemplo paradigmático es el de la soja transgénica $\mathrm{rr}^{5}$ y el glifosato de la mano de la siembra directa, la combinación de una semilla de laboratorio que es resistente a un herbicida específico, ambos producidos por la misma empresa transnacional (en este caso la empresa Monsanto, cuya expresión rioplatense es la empresa Nidera).

Trabajando con información relativa a todo el departamento de Río Negro, ${ }^{6}$ se puede ver claramente el aumento de la superficie destinada a la producción forestal (eucalyptus y pino). Mientras entre 1975 y 1989 la superficie departamental destinada a esta producción era de 3.494 hectáreas, en 2008 la superficie total forestada llegaba a 104.217 hectáreas. ${ }^{7}$ En lo que respecta a los actores de este proceso de expansión, dos empresas concentran la mayor cantidad de superficie: Forestal Oriental (propiedad de los capitales finlandeses que también son dueños de la Planta de Celulosa de UPM/Botnia en la ciudad de Fray Bentos, también en el departamento de Río Negro) y EUFORES (propiedad hasta el año 2009 de la española ENCE, desde entonces ha pasado a manos de una asociación entre la chilena Arauco y la sueco-finlandesa Stora Enso, bajo el nombre de Montes del Plata).

En lo que respecta a la producción de soja la superficie cultivada en el departamento de Río Negro prácticamente se ha triplicado desde la zafra 2003/2004 (55.218 hectáreas) a la zafra 2009/2010 (151.812 hectáreas). ${ }^{8}$ En este rubro es más difusa la identificación de los actores detrás de la expansión del cultivo, pero las empresas que ha tenido una mayor proyección en la producción sojera en el litoral oeste de Uruguay son las empresas de capitales

\footnotetext{
5 RR es la denominación de esta semilla, precisamente por su resistencia al glifosato, o sea RoundUpnombre comercial de este producto- Ready.

6 Existen importantes dificultades para analizar procesos de transformación como este que ha sucedido en el medio rural uruguayo, entre otras cosas por la dificultar de desagregar la información disponible a la escala de un área protegida o aún de su zona de amortiguación. El último Censo General Agropecuario fue realizado en el año 2000 y precisamente ha sido en el período posterior al censo donde se han profundizado estas grandes transformaciones en el agro uruguayo. La información disponible es relativa a encuestas sectoriales realizadas por el Ministerio de Ganadería, Agricultura y Pesca, cuyos datos no pueden ser desagregados más allá de la escala departamental (que es precisamente la que usaremos aquí para reconstruir en líneas generales este proceso).

7 Información estadística de la Dirección Forestal del MGAP (www.mgap.gub.uy).

8 Datos de IICA (2009) y Paolino, Lanzilotta y Perera (2009).
}

Horizontes Antropológicos, Porto Alegre, ano 20, n. 41, p. 331-356, jan./jun. 2014 
argentinos El Tejar y Agronegocios Del Plata (Oyhantçabal; Narbondo, 2011). En este caso, además del aumento de la superficie cultivada ha sido significativo el cambio en la relación entre cultivos de invierno y cultivos de verano y una disminución de la rotación agricultura/ganadería, en favor de una secuencia agricultura/agricultura, lo que tendencialmente permite preveer una especialización agrícola de la zona:

El destino inmediato posterior a los cultivos de verano son cultivos de invierno y barbecho para futuros cultivos de verano en la próxima campaña. [...] en 2009 se cuantificó que el destino inmediato posterior a la cosecha de los cultivos de verano, fue que el $66 \%$ de la superficie se destina a cultivos de invierno 2009/2010 y 30,6\% se destina a barbecho para un próximo cultivo de verano. (Paolino; Lanzilotta; Perera, 2009, p. 25).

Se ha procesado un cambio en los actores empresariales, con protagonismo directo de conglomerados empresariales transnacionales, cambios tecnológicos que a su vez permiten (o provocan) significativos cambios en la escala de producción. Este proceso a su vez se refleja en la tenencia y de control de la tierra.

En la primera década del siglo XXI un equivalente al 52\% de la superficie total del departamento de Río Negro cambió de dueño, mientras que los arrendamientos acumulados son equivalentes al $42 \%$ de la superficie departamental. Como se puede apreciar, estos no son apenas cambios productivos, sino que afectan la estructura agraria del país, ${ }^{9}$ y profundamente la del departamento de Río Negro (Santos, 2011).

\section{Las transformaciones recientes y el impacto ambiental}

Empezando por estas últimas situaciones, las transformaciones productivas de la primera década del siglo XXI han generado un desplazamiento de la mano de obra tradicionalmente rural (peones de baja calificación) a estas

\footnotetext{
9 Según sostienen Oyhantçabal y Narbondo (2011, p. 63) en Uruguay “los "nuevos agricultores” pasaron de no existir" en 2000 "a representar el 12\% de los productores y controlar el 54\% de la superficie de los cultivos agrícolas" en 2007. Su principal rubro es el cultivo de soja, del cual controlan la mayor parte de la superficie, de modo que al 2007, de los 800 productores de soja, cerca del 1\% (11 empresas) controlaba el $37 \%$ de la superficie.
} 
pequeñas localidades, donde han basado su subsistencia en actividades de caza, pesca y recolección. Esto ha implicado un claro aumento en la presión sobre los recursos naturales, ya que ha aumentado la cantidad de personas que subsisten en relación a estas actividades.

Al mismo tiempo, como veremos a continuación, esta intensificación de la producción agrícola ha generado un aumento en el uso de agrotóxicos, lo que ha tenido su consecuente impacto ambiental, precisamente en relación a las actividades que realizan estos sectores, básicamente en lo que tiene que ver con la pesca y la apicultura (incluimos en esta amplia denominación no sólo la producción de miel con destino a la comercialización, sino a la captura de enjambres, tarea que podría colocarse en el inicio de la cadena productiva de la apicultura, con la recolección de enjambres silvestres para destinarlos a la producción).

En Uruguay no existen relevamientos directos de los impactos de la aplicación de agrotóxicos en la agricultura. Los únicos momentos en que se realizan mediciones en los niveles de estas sustancias en el ambiente tienen que ver con eventos de mortandades masivas de animales (peces, abejas y en zonas próximas en el vecino departamento de Paysandú, incluso de vacas y terneros).

Un estudio de la organización Vida Silvestre ${ }^{10}$ a lo largo del año 2009, detectó "la presencia de residuos de plaguicidas altamente tóxicos en peces de valor comercial y consumidos a nivel local” (Vida Silvestre, 2010, p. 6) como tarariras, bagres, sábalos y bogas. Con respecto a la agricultura, el estudio constató que "los insecticidas utilizados en los sistemas agrícolas estudiados, tiene un elevado impacto sobre la apicultura, generando entre otras cosas, una clara disminución de la producción de miel” (Vida Silvestre, 2010, p. 6).

En lo que respecta a los momentos en que se registraron mortandades masiva de peces y abejas, el estudio permitió constatar "altas concentraciones” de plaguicidas, así como en "suelos productivos tiempo después de su aplicación (hasta 3 años en suelos de uso forestal y un año después en suelo de uso sojero) y su presencia en suelos de ambientes naturales, incluyendo el área protegida” (Vida Silvestre, 2010, p. 6).

${ }^{10}$ El proyecto llevado adelante por Vida Silvestre contó con financiamiento de la UICN (Unión Internacional para la Conservación de la Naturaleza) e involucró a equipos de investigadores de las Facultad de Química y Ciencias de la Universidad de la República y del Instituto Nacional de Investigaciones Agropecuarias (INIA). Los análisis de las muestras fueron realizados en laboratorios de Alemania, en virtud de la dificultad de encontrar técnicas de medición ajustadas en laboratorios de la región.

Horizontes Antropológicos, Porto Alegre, ano 20, n. 41, p. 331-356, jan./jun. 2014 


\section{La acumulación por desposesión vivida desde abajo}

Los actores locales han vivido desde adentro los diferentes efectos de la intensificación de la agricultura en la zona. Primero por el aumento en el precio de la tierra y el desplazamiento de productores rurales (ya sea arrendatarios o pequeños propietarios), sustituidos por empresas transnacionales, alguna de ellas identificables -como las forestales, que señalan sus campos con carteles e incluso tienen fundaciones de vínculo con las comunidades- y otras muy difusas, como las del agronegocio.

Una de las primeras dificultades las vivieron los apicultores. Por su sistema productivo, se puede decir que su producción es trashumante: las colmenas se mueven y se ubican en diferentes lugares. Por lo general, los apicultores no son dueños de la tierra, por lo que dependen de conocimiento previo o vínculos de confianza para acceder a los lugares dónde colocar sus colmenas. En el caso de la forestación, este vínculo se ha institucionalizado al punto de tener que pagar un arrendamiento (por colmena) para poder acceder al derecho de colocar las colmenas dentro de las plantaciones forestales.

Aquí reside uno de los motivos principales al hecho de porqué los apicultores en general no han hecho públicas las denuncias de mortandad de abejas que se vienen registrando desde que se ha intensificado el uso de agrotóxicos en la zona, a través de las fumigaciones para la agricultura (básicamente, para la soja, sobre todo y en niveles nunca antes conocidos, desde el 2003). Los apicultores se ven enfrentados al dilema de no denunciar la mortandad de abejas para no se expulsados del lugar donde les han permitido instalar sus colmenas o perder el lugar donde se encuentran por un reclamo de justicia productiva, antes que ambiental. Aquí juega mucho la relación entre los apicultores y los responsables de los campos o aún de los propietarios que arriendan para la soja (y a quienes indirectamente perjudicarían, al realizar la denuncia).

En el caso de los pescadores, hay una percepción directa del aumento de las mortandades de peces. Sin embargo no es directa la asociación -o por lo menos, no lo era hasta la presentación del estudio sobre impactos de los agrotóxicos en la región- entre estos fenómenos y la intensificación de la producción agrícola.

Por ello, seguramente, el conflicto entre actividades como la pesca o la apicultura y el agronegocio ha tenido hasta ahora mucho de silencioso, y sólo 
recientemente -con la presentación del estudio de Vida Silvestre- se ha hecho público.

Algunos de los pescadores de Nuevo Berlín coloca el problema de la contaminación de los peces en clave de una situación de subsistencia de toda la población local y de la de su propia familia, "ya no sé para dónde disparar" era una frase recurrente, no sólo en referencia al lugar sino a la búsqueda de fuentes alimenticias seguras.

Por su parte en alguno de los discursos de los apicultores entra en juego la existencia del área protegida, en un carácter instrumental, que reafirma la defensa de su propio punto de vista:

La verdad es que la situación es desesperante para los apicultores; se dice que en Uruguay se ha perdido la mitad de las colmenas, y acá tenemos un área protegida se da una contradicción por la mortandad masiva de abejas que hemos tenido. Queremos que el gobierno tome carta en el asunto rápidamente, los apicultores pensamos y analizamos que si el gobierno no realiza una acción rápida, el sector se termina en cuatro o cinco años en la zona. No pretendemos que el agro se corte ni que no se siembre más soja, pero sí que haya un control muy estricto. (Muñoz, 2010, p. 9).

Pero, ¿hasta dónde este es un conflicto redistributivo ambiental? ¿Podemos hablar aquí de demandas de justicia ambiental o estamos ante un conflicto “productivo”? Más allá de los argumentos en juego, es claro que hay un grupo de actores (básicamente pescadores y apicultores, con fundamentos a partir de su vínculo con una organización ambientalista) que colocan este conflicto en términos de redistribución de la contaminación poniendo en cuestión los impactos de la utilización incontrolada de agrotóxicos.

\section{Naturaleza, sustentabilidad y conflictos económicos}

La manera de percibir y representar las relaciones entre la sociedad y la naturaleza es producto de procesos históricos y sociales y por ello, la manera en que el ser humano se ha pensado y representado en relación con la naturaleza es diversa a lo largo de la historia y a través de los diferentes contextos sociales. Cada grupo humano (cada sociedad, cada cultura) tiene una particular percepción de un entorno inmediato que -en muchas ocasiones- es definido como "natural” aunque sea producto de modificaciones antrópicas también

Horizontes Antropológicos, Porto Alegre, ano 20, n. 41, p. 331-356, jan./jun. 2014 
históricas y acumulativas. En cierto modo, lo que se defina por conservación y por producción dependerá del contexto de significación en que esta idea esté inscripta, o sea, dependerá de cómo se plantee la relación entre naturaleza y sociedad o entre cultura y ambiente. Es así que debemos enfocarnos en lo que se define como natural o naturaleza en cada momento histórico y en cada lugar geográfico para discutir en ese contexto si el hombre está "dentro o fuera” de esa noción de naturaleza (West; Igoe; Brockington, 2006).

La idea de área protegida, heredera de la noción de "parque” implica una posición externa, una posición de observador, de admirador de una naturaleza que está fuera de ella. Pero muchas veces esa naturaleza o esos paisajes propios de los parques no son un producto solamente natural ya que, por ejemplo, la vegetación ha sufrido importantes transformaciones producto de la intervención humana. Por otra parte, la idea de establecer un área que se debe proteger también implica una cierta concepción que denota el carácter externo del ser humano con respecto a la naturaleza, ya que se plantea la responsabilidad de los seres humanos como tales de conservar o proteger un determinado espacio, sin "intervenir" como postulan muchos exponentes del conservacionismo.

En un principio la coexistencia entre conservación y producción se consideraba a partir de criterios de ordenamiento territorial: era necesario establecer la áreas de exclusión en las cuáles solamente se iban a realizar actividades de conservación. Un resguardo del avance de la frontera agrícola, una especie de generación de islas de naturaleza en un campo de avanzada modificación humana. Pero a partir de la segunda mitad del Siglo XX, la idea de Parques Naturales como santuarios se fue transformando y complejizando, tendiendo a una idea que incluye a la conservación como estrategia de desarrollo sustentable y, al mismo tiempo, a la visualización de determinadas prácticas productivas (que podríamos llamar de amigables con el ambiente) como otras formas de conservación. Los datos analizados para el departamento de Río Negro y particularmente el entorno del área protegida Esteros de Farrapos e Islas del Río Uruguay claramente cuestionan esta posible coexistencia. El avance de los sistemas productivos del agronegocio implica el proceso de extracción de riqueza a partir de los recursos naturales conduciendo al agotamiento de recursos tales como el suelo o a la degradación de recursos como el agua y el aire a partir del uso de agrotóxicos, que por otra parte son los medios de vida de la mayor parte de la población. 


\section{Naturalismos}

Existen muchos fundamentos para abordar desde las ciencias sociales las políticas, discursos y prácticas de la "conservación de la naturaleza”. Procederemos aquí a plantear algunas de ellas. En el plano teórico/epistemológico, las ideas de conservación de la naturaleza son una de las expresiones centrales del pensamiento moderno en relación a lo que provisoriamente podemos llamar “lo natural”. Como ha planteado Philippe Descola (2004, p. 88, traducción mía), la noción de naturaleza es construida socialmente y su visión dicotómica en relación a lo social es propia de la cosmología occidental, que ha construido una suerte de fetichización de la naturaleza:

Típico de las cosmologías occidentales desde Platón y Aristóteles, el naturalismo creó un dominio ontológico específico, un lugar de orden y necesidad, donde nada sucede sin una razón o una causa, ya estén originadas éstas en Dios [...] o sean inmanentes a la fábrica del mundo ('las leyes de la naturaleza'). ${ }^{11}$

Este modo de identificación que Descola (2004, p. 88, traducción mía) reconoce como "naturalismo" es "la creencia de que la naturaleza simplemente existe, y de que algunas cosas deben su existencia y desarrollo a un principio ajeno tanto al azar como los efectos de la voluntad humana” y que actúa como un supuesto que estructura nuestra epistemología. ${ }^{12}$

Este naturalismo tiene dos expresiones en la relación entre la sociedad y la naturaleza en la cosmología occidental y moderna. Una es el naturalismo depredador y otra el naturalismo conservacionista.

En cuanto al naturalismo depredador, es menos un valor que una práctica de la vieja Europa, nacida en la Edad Media cuando grandes extensiones de bosques fueron despejadas para cultivar; una práctica que adquirió legitimidad con la filosofía cartesiana, y su expresión plena con la mecanización del mundo, tanto en lo físico como en el sentido técnico de la expresión; una práctica que se

11 En el original: “Typical of western cosmologies since Plato and Aristotle, naturalism creates a specific ontological domain, a place of order and necessity where nothing happens without a reason or a cause, whether originating in God [...] or immanent to the fabric of the world ('the laws of nature')."

12 En el original: "Naturalism is simply the belief that nature does exist, that certain things owe their existence and development to a principle extraneous both to chance and to the effects of human will.”

Horizontes Antropológicos, Porto Alegre, ano 20, n. 41, p. 331-356, jan./jun. 2014 
transformaría en el destino histórico de Europa, bajo el nombre de producción, cuando la sociedad burguesa se las arregló para autoconcebirse como la encarnación de un orden natural. (Descola, 2004, p. 97, traducción mía). ${ }^{13}$

En este sentido, la protección de la naturaleza no es más que la contracara de esta relación de depredación con el entorno:

en los movimientos conservacionistas contemporáneos, la protección de los no humanos no está desprovista de autogratificación. Se transfiere el dominio cartesiano y la propiedad de la naturaleza a otro plano, un pequeño enclave en donde se alivia la culpa y la dominación paternalista eufemísticamente transmutado en la preservación protectora y la apreciación estética. (Descola, 2004, p. 91, traducción mía). ${ }^{14}$

Desde este punto de vista, procesos de depredación ambiental y de conservación de la vida silvestre responden a una misma lógica: una lógica que ubica a la sociedad por fuera de la naturaleza. Esto es algo que también a advertido Ingold (2002), en relación a la utilización de los conceptos de naturaleza y ambiente.

La distinción entre ambiente y naturaleza corresponde a la diferencia de perspectiva entre vernos a nosotros mismos como seres dentro del mundo y como seres fuera de él. Por otra parte, tendemos a pensar la naturaleza como externa, no sólo a la humanidad [...] sino también externo a la historia, como si el mundo natural proveyera un telón de fondo permanente para la realización de los asuntos humanos. (Ingold, 2002, p. 20, traducción mía). ${ }^{15}$

${ }^{13}$ En el original: "As for predatory naturalism, it is less a value than an old European practice, born in the Middle Ages when large tracts of forest where cleared for cultivation; a practice which acquired its legitimacy with Cartesian philosophy, and its full expression with the mechanisation of the world-in the physical as well as in the technical sense of the expression; a practice which then transformed into the historical destiny of Europe, under the name of production, when bourgeois society managed to conceive itself as the embodiment of a natural order."

${ }^{14}$ En el original: "in contemporary conservationist movements, the protection of non-humans is not devoid of selfgratification. It transfers the Cartesian mastery and ownership of nature to another plane, a small enclave where guilt is alleviated and domination euphemistically transmuted into patronising preservation and aesthetic entertainment."

15 En el original: "Thus the distinction between environment and nature corresponds to the difference in perspective between seeing ourselves as beings within a world and as beings without it. Moreover we tend to think of nature as external not only to humanity, as I have already observed, but also to history, as though the natural world provided an enduring backdrop to the conduct of human affairs.”

Horizontes Antropológicos, Porto Alegre, ano 20, n. 41, p. 331-356, jan./jun. 2014 


\section{El metabolismo sociedad-naturaleza}

Un concepto que es útil para trabajar la relación sociedad-naturaleza desde la discusión en torno a la sustentabilidad es el de metabolismo que aparece en Marx (desarrollado por Foster, 2000). Esta noción de metabolismo está anclada directamente en la idea de trabajo, que en el pensamiento de Marx (1987, p. 215) es la mediación entre la sociedad y la naturaleza:

el trabajo es un proceso entre el hombre y la naturaleza, un proceso en que el hombre media, regula y controla su metabolismo con la naturaleza. El hombre se enfrenta a la materia natural misma como un poder natural. Pone en movimiento las fuerzas naturales que pertenecen a su corporeidad, brazos y piernas, cabeza y manos, a fin de apoderarse de los materiales de la naturaleza bajo una forma útil para su propia vida. Al operar por medio de ese movimiento sobre la naturaleza exterior a él y transformarla, transforma a la vez su propia naturaleza.

En el caso de Farrapos, es claro que el acceso a la tierra es uno de los factores determinantes en el proceso de exclusión de los trabajadores rurales, así como de los pequeños productores familiares (arrendatarios o propietarios, aunque éstos últimos se vean en parte beneficiados del proceso). Esta es la contracara del proceso de concentración de la tierra.

Pero la discusión instalada a partir de la afectación de los sistemas productivos que dependen en mucho mayor grado de la calidad ambiental (la pesca, la apicultura) tiene que ver con las formas de apropiación/expropiación en relación al bien común que podemos denominar provisoriamente ambiente.

El acceso al agua y al aire sin contaminación, la posibilidad de que los servicios ambientales (polinización, el cumplimiento del ciclo reproductivo de peces y abejas, en este caso) se lleven a cabo son factores críticos, y aunque nadie se apropie directamente de ellos, si podemos dar cuenta de procesos de acumulación por desposesión, como los caracteriza Harvey. Dentro de estos procesos se encuentra

La reciente depredación de los bienes ambientales globales (tierra, aire, agua) y la proliferación de la degradación ambiental, que impide cualquier cosa menos los modos capital-intensivos de producción agrícola, han resultado de la total transformación de la naturaleza en mercancía. (Harvey, 2007, p. 114).

Horizontes Antropológicos, Porto Alegre, ano 20, n. 41, p. 331-356, jan./jun. 2014 
En cierto sentido podemos pensar, a partir de los planteos de Tilly (2000) hasta dónde un proceso como la expansión del agronegocio en un territorio concreto no genera una forma de desigualdad categorial en relación a la posesión o no de un recurso básico para la reproducción social, como es la tierra. Una visión que incluyera los impactos de este tipo de sistemas productivas nos obligaría a ampliar esa idea de tierra como recurso a la tierra como sustento de procesos ecosistémicos, lo que nos llevaría a incluir al agua, el aire o el hábitat de especies fundamentales para la las dinámicas ecológicas pero al mismo tiempo fundamentales para la reproducción social. El mecanismo de exclusión podría operar en relación al criterio básico (acceso o no a la tierra en tanto que recurso) mientras que el acaparamiento de oportunidades se marca en la imposibilidad de coexistencia de estos sistemas productivos -en un contexto de escasa regulación- con otros (como por ejemplo, los que hemos discutido aquí de la pesca o la apicultura). En la situación de acceso a la tierra, es necesario tomar en cuenta lo novedoso de la expansión de la soja, en relación a las amplias superficies de tierra que son controladas no por la vía de la propiedad, sino por la del arrendamiento. Si bien la exclusión operaría a través del mercado, no pasa necesariamente por la propiedad de la tierra, sino por la capacidad de tener o captar recursos hacia el pago del arrendamiento de la tierra. Una nueva modalidad, en el marco de un viejo mecanismo de diferenciación social en el campo.

\section{La escala global de la reproducción social: apuntes sobre naturaleza y sociedad}

Los datos que hemos analizado para el departamento de Río Negro y particularmente el entorno del Área protegida Esteros de Farrapos e Islas del Río Uruguay claramente confirman la tendencia general de la producción en un marco capitalista, que es, necesariamente, la degradación de los recursos naturales. El acaparamiento de tierras -a través del avance del agronegocioimplica el proceso de extracción de riqueza a partir de los recursos naturales conduciendo -como quizás no había sucedido antes- al agotamiento de recursos tales como el suelo o a la degradación de recursos como el agua y el aire a partir del uso de agrotóxicos. Esto ocurre en un espacio que también es objeto de políticas globales de conservación, como la Convención RAMSAR o la Convención sobre Diversidad Biológica de Naciones Unidas. 
En el plano local, los usos de los recursos naturales que los habitantes del área realizan para su reproducción social nos plantean un debate aún mayor: ¿que pasa si consideramos esas actividades de subsistencia (caza, pesca, recolección de enjambres de abejas) como actividades "productivas"? en tanto transformación de la naturaleza que viabiliza una determinada reproducción social (Narotzky, 2004). En este sentido sí podríamos plantear una coexistencia entre conservación y producción, pero evidentemente estas dinámicas no son las hegemónicas y deben ser evaluadas en su propio contexto. Las transformaciones en el uso y propiedad de la tierra han determinado que un número mayor de personas resida en las zonas urbanas, despojadas de sus habituales medios de producción y reproducción. Por ende, la presión sobre las actividades de subsistencia también ha aumentado y su carácter no antagónico con la conservación.

Esta situación anula la premisa de Marx acerca del metabolismo de la sociedad con la naturaleza, según la cual lo que requiere explicación

no es la unidad del ser humano con la naturaleza, esto es parte de la naturaleza física y química. Lo que requiere explicación es el proceso histórico a través del cual se separa -se aliena- la existencia humana de las condiciones naturales para reproducirse. (Foladori, 2001, p. 77).

Parece claro entonces que la generación de áreas protegidas de ningún modo supone límites al avance de la expansión del capitalismo sobre el campo, sobre la naturaleza. La preocupación que viven los actores locales de las áreas cobran mayor vigencia en este contexto de discusión: ¿por qué se prohíben actividades extractivas de subsistencia y a pocos metros de distancia el agronegocio de desarrolla a escalas históricas nunca antes registradas generando una serie de beneficios que no son distribuidos, por lo menos en el nivel local?

Evidentemente la tensión conservación/producción no será resuelta en el entorno del Área protegida Esteros de Farrapos e Islas del Río Uruguay. Será necesario que la implementación de esta política pública de conservación tome en cuenta las dinámicas propias de las poblaciones afincadas en el espacio que ocupará el área así como los procesos socioeconómicos que se vienen registrando en la región, para poder minimizar los conflictos que se producirán a la hora de tratar de acompasar la protección ambiental con la generación de riqueza a partir de la explotación de los recursos naturales. 
En cierto sentido, la idea de naturaleza que orienta la implementación de áreas protegidas parece tener mucho más en común con la visión desde arriba que tiene el agronegocio. No es otra cosa que lo que ha planteado Horacio Machado Araóz; una ecología política de la modernidad debe desentrañar la articulación Ciencia-Estado-Capital con el fin de desnaturalizar la naturaleza (Machado Araóz, 2009).

Las diferentes territorialidades en juego se evidencian en las prácticas de los actores y en sus discursos, discursos que como se redefinen los conceptos de pertenencia y ciudadanía en la poscolonialidad. En este marco, también es crítico entender si el establecimiento de áreas protegidas forma parte de una estrategia de mitigación de los impactos del desarrollo o de la superación de las limitantes que el avance de las relaciones capitalistas de producción genera sobre la relación sociedad-naturaleza. En este sentido, la idea de superación de las nociones tradicionales de desarrollo -aún las de desarrollo sustentabledebe ser contemplada. Esto es lo que Arturo Escobar (1996) ha denominado posdesarrollo.

Entendida como parte del proceso de globalización, la expansión de las áreas protegidas es la contracara de la expansión del capitalismo sobre los bienes de la naturaleza. La institucionalidad ambiental transnacional y multiescalar es el contrapeso de la consolidación del agronegocio en el campo -en lugar de la producción campesina y de la producción familiar- y del avance de industrias extractivas sobre la naturaleza (como la minería o aún la propia intensificación de procesos de producción agrícola).

A pesar del aumento sostenido en la superficie bajo condiciones de protección, de ninguna manera esto implica la consolidación de contratendencias a la tendencia general de la producción en un marco capitalista que es, necesariamente, la degradación de los recursos naturales. Si se alcanzase la meta de asegurar la protección de el $12 \%$ de la superficie del plantea, en el casi 90\% restante del planeta las lógicas de producción capitalista serán las predominantes, las impulsadas por las dinámicas de avances de la frontera agrícola y de intensificación del sistema industrial de producción aplicado a la agricultura. 


\section{Referencias}

BOLETÍN SNAP. Montevideo, n. 6, otoño 2010.

BORRAS Jr., S.; FRANCO, J. La política del acaparamiento mundial de tierras: Replanteando las cuestiones de tierras, redefiniendo la resistencia. Rotterdam: TNI; LDPI; ICAS; ICCO, 2010. (ICAS Working Paper Series, n. 001). Disponible en: http://www.tni.org/sites/www.tni.org/files/La\%20 pol\%C3\%ADtica\%20del\%20acaparamiento\%20mundial\%20de\%20tierras. pdf>. Acceso: 2 nov 2011.

DESCOLA, P. Constructing natures: symbolic ecology and social practice. In: DESCOLA, P.; PÁLSSON, G. (Comp.). Nature and society: anthropological perspectivas. London: Routledge, 2004. p. 82-102.

DGRNR-MGAP. Ficha informativa Ramsar Esteros de Farrapos e Islas del Río Uruguay. Montevideo: Ministerio de Ganadería, Agricultura y Pesca, República Oriental del Uruguay, 2004.

DGRNR-MGAP. Informe nacional sobre la aplicación de la Convención de Ramsar sobre los humedales. Presentado a la $10^{\text {a }}$ Reunión de la Conferencia de las Partes Contratantes, República de Corea, 2008.

DIEGUES, A. C. El mito moderno de la naturaleza intocada. São Paulo: Núcleo de Apoio à Pesquisa sobre Populações Humanas e Áreas Úmidas Brasileiras/Universidade de São Paulo, 2005.

DOWIE, M. Los refugiados del conservacionismo. Cuando la conservación implica desterrar a la gente. Revista Biodiversidad, sustento y culturas, Montevideo, n. 49, p. 1-6, 2006.

ESCOBAR, A. La invención del Tercer Mundo: construcción y reconstrucción del desarrollo. Bogotá: Norma, 1996.

FOLADORI, G. El metabolismo con la naturaleza. Herramienta, Buenos Aires, año 6, n. 16, p. 75-88, 2001.

FOSTER, J. La ecología de Marx: materialismo y naturaleza. Barcelona: El Viejo Topo, 2000. 
HARVEY, D. El nuevo imperialismo. Buenos Aires: Akal, 2007.

HERNÁNDEZ, V. La ruralidad globalizada y el paradigma del agronegocio en las pampas gringas. In: GRAS, C.; HERNÁNDEZ, V. (Coord.). La Argentina rural: de la agricultura familiar a los agronegocios. Buenos Aires: Biblos, 2009. p. 15-37.

IICA. Evolución y situación de la cadena agroalimentaria sojera. Montevideo, 2009.

INGOLD, T. The perception of the environment. London: Routledge, 2002.

MACADAR D.; DOMÍNGUEZ, P. Migración interna. In: VARELA PETITO, C. (Coord.). Demografía de una sociedad en transición: la población uruguaya a inicios del Siglo XXI. Montevideo: UNPFA; Trilce, 2008. p. 83-113.

MACHADO ARÁOZ, H. Ecología política de la modernidad. Una mirada desde Nuestra América. In: CONGRESO ALAS, 27., Buenos Aires, 2009. Anales... Buenos Aires: Asociación Latinoamericana de Sociología, 2009. p. 1-13.

MARX, K. El capital: tomo I: El proceso de producción del capital. México: Siglo XXI, 1987.

MUÑOZ, A. Jaque: apicultores solicitan que el gobierno mejore el control de agrotóxicos. La Diaria, Montevideo, p. 9, 23 jun. 2010.

NAROTZKY, S. Antropología económica: nuevas tendencias. Barcelona: Melusina, 2004.

NUESTRA TIERRA. Río Negro. Montevideo: Editorial Nuestra Tierra, 1970. (Colección Los departamentos, n. 6)

OYHANTÇABAL, G.; NARBONDO, I. Radiografía del agronegocio sojero: descripción de los principales actores y los impactos socioeconómicos en Uruguay. Montevideo: REDES-AT, 2011.

PAOLINO, C.; LANZILOTTA, B.; PERERA, M. Tendencias productivas en Uruguay: los sectores agroindustriales, turismo y minería. Montevideo: Proyecto SNAP, 2009. 
SANTOS, C. ¿Qué protegen las áreas protegidas?: conservación, producción, Estado y sociedad en la implementación del Sistema Nacional de Áreas Protegidas. Montevideo: Trilce, 2011.

THE WORLD BANK. Rising global interest in farmland: can it yield sustainable and equitable benefits? Washington, 2010. Disponible en: <http:// farmlandgrab.org/wp-content/uploads/2010/09/ESW_Sept7_final_final.pdf >. Acceso: 2 nov. 2011.

TILLY, C. La desigualdad persistente. Buenos Aires: Manantial, 2000.

VIDA SILVESTRE. Plaguicidas en la cuenca del Parque Nacional Esteros de Farrapos e Islas del Río Uruguay: resumen ejecutivo. Montevideo, 2010.

WEST, P.; IGOE, J.; BROCKINGTON, D. Parks and peoples: the social impact of protected areas. Annual Review of Anthropology, Palo Alto, v. 35, p. 251-277, 2006.

Recebido em: 27/08/2013

Aprovado em: 19/12/2013 